\title{
Application du procédé séchage-friture aux amandes de karité : cinétique de séchage
}

\author{
Hilaire Macaire WOMENI ${ }^{1}$ \\ Robert NDJOUENKEU ${ }^{2}$ \\ César KAPSEU ${ }^{2}$ \\ Michel PARMENTIER ${ }^{3}$ \\ ${ }^{1}$ Département de biochimie, \\ Faculté des sciences, \\ Université de Dschang, \\ BP 67 Dschang /Cameroun \\ <womeni@yahoo.fr> \\ ${ }^{2}$ Ecole nationale supérieure \\ des sciences agro-industrielles (ENSAI), \\ Université de Ngaoundéré, BP 455 Ngaoundéré, \\ Cameroun \\ ${ }^{3}$ Laboratoire de physico-chimie et génie \\ alimentaires, ENSAIA - INPL, \\ 2, avenue de la Forêt de Haye, \\ 54500 Vandœuvre-les-Nancy cedex, France
}

\begin{abstract}
Fresh kernel of shea (Butyrospermum parkii (G. Don) Kotschy) have been dehydrated by a deep-fat frying dehydration process with view to extract subsequently the shea butter. The deep-fatfrying dehydration significantly reduces the water content of the shea kernels within a very short period of time. The effects of some operating parameters on the mass transfers during deep-frying showed that the rate of drying was linked to the temperature of the bath, the frying time, the thickness of the kernels, and the mass ratio kernels/butter. A final moisture of about $5 \%$ was obtained after 10 minutes of frying at $150-180{ }^{\circ} \mathrm{C}$ applied to kernels cut into slices of 2 to $6 \mathrm{~mm}$ thick with a weight ratio kernels/butter of less than 0.04 . The residual moisture content can be expressed using mathematical models taking into account the operating parameters. A negative linear correlation was fund between the water and butter contents. Such a rapid frying was considered to improve the quality of the fat extracted from shea kernels that have been preserved from moisture degradation during processing.
\end{abstract}

Key words: Butyrospermum parkii, shea butter, deep-fat frying dehydration, kinetic of drying

Le karité (Butyrospermum parkii (G. Don) Kotschy) est un arbre africain de la famille des Sapotacées, typique des savanes arborées soudaniennes, surtout connu par le beurre extrait de ses amandes, d'où l'appellation d'arbre à beurre. Les procédés d'extraction de cette matière grasse comprennent certaines opérations unitaires critiques pour la qualité du beurre, comme l'a montré une analyse des procédés traditionnels d'extraction suivant un référentiel HACCP [1] : le dépulpage des fruits, le décorticage et la cuisson des graines, le séchage et le broyage des amandes, l'extraction proprement dite. Le séchage des amandes se fait généralement au soleil ou par fumage. Or, les amandes ayant une teneur initiale en eau élevée, elles offrent des conditions favorables à l'activité des enzymes de dégradation comme celles des micro-organismes présents. Par conséquent, les techniques lentes de déshydratation des oléagineux conduisent à des huiles dont la qualité est souvent dégradée [2]. L'utilisation du séchage par friture avant l'extraction d'huile réduit rapidement la teneur en eau et limite les risques de développement microbiologiques, de pollution et de dégradations chimiques comme l'oxydation. De plus, la mise en œuvre de cette technique est simple, et son coût global (main-d'œuvre et énergie) plutôt faible [3, 4]. Si la friture est depuis longtemps connue comme mode de cuisson des aliments, son utilisation comme moyen de déshydratation est plus récente. La déshydratation des produits amylacées a été étudiée en relation avec les variables du procédé, notamment ceux à base de pommes [5-8], de la pâte de maïs $[9,10]$, de plantain [11, 12] de patate [13] ou de manioc [14-16]. Les principales applications du séchage par friture de produits à la fois riches en eau et en matières grasses, sont le séchage de la noix de coco et celui des déchets d'équarrissage. Les travaux de Merion [17] ont montré que ce procédé pouvait également être appliqué à la pulpe d'avocat. Cette technique a permis l'amélioration de la qualité de I'huile de coco, dont l'extraction était handicapée par la qualité du coprah obtenu par séchage solaire des tranches d'amandes de coco [18]. L'utilisation de cette technique pour déshydrater les amandes de karité permettrait en peu de temps de ramener la teneur en eau de plus de $50 \%$ à une valeur de 10-15\%, optimale pour l'extraction du beurre [19]. Les investigations menées sur le procédé de friture ont révélé que la température du bain et le temps d'immersion des produits sont les principales variables de conduite dont dépendent le transfert de masse [20] et les transformations et réactions secondaires dans les produits [6]. L'objectif du présent travail est d'étudier l'intérêt de l'application de la technique de séchage-friture aux amandes de karité par le suivi de la cinétique de séchage en fonction des variables de commande et la détermination des modèles d'expression de la teneur en eau en fonction du temps. 


\section{Matériel et méthodes}

\section{Les fruits du karité}

Les fruits de karité utilisés ont été achetés sur le marché local (Ngaoundéré, Cameroun). Ces fruits avaient une masse de $69 \pm 22 \mathrm{~g}$ et contenaient une à trois graines (noix) protégées par une coque dure d'un millimètre d'épaisseur environ, remplie par une amande de masse $21,63 \pm 6,80 \mathrm{~g}$, de longueur $40,84 \pm 51 \mathrm{~mm}$ et de diamètre $27,25 \pm 4,22 \mathrm{~mm}$ pour le petit axe et $31,56 \pm 3,57 \mathrm{~mm}$ pour le grand axe. La teneur en eau initiale est de 56,24 $\pm 2,74 \%$.

\section{Le beurre de karité}

Les essais de friture ont été réalisés dans du beurre de karité acheté auprès des paysans. La masse volumique, les indices d'iode, de saponification, d'acide et de peroxyde ont été déterminés par les méthodes normées AFNOR [21] (tableau 1).

\section{La friteuse}

La friture a été conduite dans une friteuse ménagère de marque Kenwood à régulateur de température, conçue pour fonctionner avec 2-2,5 litres d'huile.

\section{L'opération de friture}

La première phase a consisté à suivre l'effet de la température $(120,140$, 160 et $180^{\circ} \mathrm{C}$ ) et du temps de friture sur la cinétique de perte $\mathrm{d}^{\prime}$ eau et $d^{\prime}$ 'absorption d'huile d'amandes entières fraîches dans un rapport massique amande/beurre de 1/15 (120 g d'amandes pour $1800 \mathrm{~g}$ de beurre). La seconde phase a consisté à suivre, selon un plan d'expériences composite centré, la variation de la teneur en eau des amandes en considérant, outre la température et le temps de friture, l'épaisseur des amandes et le rapport massique amande/beurre. Les valeurs réelles et codées de ces 4 variables de commande sont données dans le tableau 2. Ce plan permet de calculer la teneur en eau $Y$ suivant l'équation du modèle :

$$
\begin{gathered}
Y=I+a x_{1}+b x_{2}+c x_{3}+d x_{4}+e x_{1}^{2}+f x_{1} x_{2}+g x_{1} x_{3}+ \\
h x_{1} x_{4}+i x_{2}^{2}+j x_{2} x_{3}+k x_{2} x_{4}+\mathrm{Ix}_{3}{ }^{2}+\mathrm{mx}_{3} \mathrm{x}_{4}+\mathrm{nx}_{4}{ }^{2}
\end{gathered}
$$

l, $a, b, c, d, e, f, g, h, i, j, k, l, m$ et $n$ étant les coefficients de l'équation. En fin de friture, les amandes sont extraites du bain et placées immédiatement sur du papier absorbant afin d'éliminer l'huile superficielle. Les amandes frites et refroidies à température ambiante sont ensuite pesées et leur teneur en eau et en huile après friture sont déterminées.

Tableau 1. Caractéristiques du beurre de karité utilisé pour la friture.

\begin{tabular}{|lc|}
\hline Caractéristiques & Valeurs \\
\hline Masse volumique $(\mathrm{g} / \mathrm{ml})$ & $0,92 \pm 0,04$ \\
Indice de saponification & $207,52 \pm 7,78$ \\
Indice d'iode & $89,46 \pm 3,10$ \\
Indice d'acide & $7,56 \pm 1,68$ \\
Indice de peroxyde (meq/kg) & $5,48 \pm 0,16$ \\
\hline
\end{tabular}

\begin{tabular}{|c|c|c|c|c|c|c|c|c|}
\hline \multirow[b]{3}{*}{ Essais } & \multicolumn{4}{|c|}{ Valeurs codées } & \multicolumn{4}{|c|}{ Valeurs réelles } \\
\hline & $\begin{array}{c}\text { Epaisseur } \\
\text { des amandes }\end{array}$ & $\begin{array}{l}\text { Température } \\
\text { de séchage }\end{array}$ & $\begin{array}{l}\text { Rapport massique } \\
\text { (amandes/ beurre) }\end{array}$ & $\begin{array}{c}\text { Temps } \\
\text { de séchage }\end{array}$ & $\begin{array}{c}\text { Epaisseur } \\
\text { des amandes } \\
(\mathrm{mm})\end{array}$ & $\begin{array}{c}\text { Température } \\
\text { de séchage } \\
\left({ }^{\circ} \mathrm{C}\right)\end{array}$ & $\begin{array}{l}\text { Rapport massique } \\
\text { (amandes/ beurre) }\end{array}$ & $\begin{array}{c}\text { Temps de } \\
\text { séchage } \\
\text { (minutes) }\end{array}$ \\
\hline & $x_{1}$ & $x_{2}$ & $x_{3}$ & $x_{4}$ & $x_{1}$ & $x_{2}$ & $x_{3}$ & $x_{4}$ \\
\hline 1 & 0 & 0 & 0 & 0 & 10 & 150 & 0,05 & 6 \\
\hline 2 & 0 & 0 & 0 & 0 & 10 & 150 & 0,05 & 6 \\
\hline 3 & 1 & 1 & 1 & 1 & 14 & 165 & 0,06 & 8,8 \\
\hline 4 & 1 & 1 & 1 & -1 & 14 & 165 & 0,06 & 3,2 \\
\hline 5 & 1 & 1 & -1 & 1 & 14 & 165 & 0,04 & 8,8 \\
\hline 6 & 1 & 1 & -1 & -1 & 14 & 165 & 0,04 & 3,2 \\
\hline 7 & 1 & -1 & 1 & 1 & 14 & 135 & 0,06 & 8,8 \\
\hline 8 & 1 & -1 & 1 & -1 & 14 & 135 & 0,06 & 3,2 \\
\hline 9 & 1 & -1 & -1 & 1 & 14 & 135 & 0,04 & 8,8 \\
\hline 10 & 1 & -1 & -1 & -1 & 14 & 135 & 0,04 & 3,2 \\
\hline 11 & -1 & 1 & 1 & 1 & 6 & 165 & 0,06 & 8,8 \\
\hline 12 & -1 & 1 & 1 & -1 & 6 & 165 & 0,06 & 3,2 \\
\hline 13 & -1 & 1 & -1 & 1 & 6 & 165 & 0,04 & 8,8 \\
\hline 14 & -1 & 1 & -1 & -1 & 6 & 165 & 0,04 & 3,2 \\
\hline 15 & -1 & -1 & 1 & 1 & 6 & 135 & 0,06 & 8,8 \\
\hline 16 & -1 & -1 & 1 & -1 & 6 & 135 & 0,06 & 3,2 \\
\hline 17 & -1 & -1 & -1 & 1 & 6 & 135 & 0,04 & 8,8 \\
\hline 18 & -1 & -1 & -1 & -1 & 6 & 135 & 0,04 & 3,2 \\
\hline 19 & $\alpha$ & 0 & 0 & 0 & 18 & 150 & 0,05 & 6 \\
\hline 20 & $-\alpha$ & 0 & 0 & 0 & 2 & 150 & 0,05 & 6 \\
\hline 21 & 0 & $\alpha$ & 0 & 0 & 10 & 180 & 0,05 & 6 \\
\hline 22 & 0 & $-\alpha$ & 0 & 0 & 10 & 120 & 0,05 & 6 \\
\hline 23 & 0 & 0 & $\alpha$ & 0 & 10 & 150 & 0,07 & 6 \\
\hline 24 & 0 & 0 & $-\alpha$ & 0 & 10 & 150 & 0,03 & 6 \\
\hline 25 & 0 & 0 & 0 & $\alpha$ & 10 & 150 & 0,05 & 11,6 \\
\hline 26 & 0 & 0 & 0 & $-\alpha$ & 10 & 150 & 0,05 & 0,4 \\
\hline
\end{tabular}

Tableau 2. Valeurs codées et réelles du plan d'expériences composite centré. 


\section{Expression des résultats}

- Teneur en eau: la teneur en eau des amandes frites, notée TE, est déterminée par étuvage à $105^{\circ} \mathrm{C}$ pendant 24 heures. Elle est calculée par rapport à la base humide et s'exprime en grammes pour $100 \mathrm{~g}$ de produit initial :

$$
\mathrm{TE}=\left(\mathrm{m}_{0}-\mathrm{m}_{\mathrm{et}}\right) * 100 / \mathrm{m}_{0}(\%)
$$

Où $\mathrm{m}_{0}=$ masse de l'échantillon avant séchage à l'étuve et $\mathrm{m}_{\mathrm{et}}=$ masse de l'échantillon après séchage à l'étuve.

- Vitesse de perte d'eau. En effectuant notre essai de friture, nous avons une masse $m_{0}$ à sécher. Après un temps $t_{1}$ de friture, nous obtenons une masse $m_{1}$. La vitesse de séchage $V$ (en g par minute) est donnée par :

$$
V=m_{0}-m_{1} / t_{1}-t_{0}
$$

- Teneur en huile. La teneur en huile $\left(\mathrm{TH}_{\mathrm{BS}}\right)$, est exprimée en gramme pour $100 \mathrm{~g}$ de matière sèche. La détermination de la teneur en huile est effectuée par extraction au soxhlet à l'éther de pétrole pendant 10 heures [22].

$$
\mathrm{TH}_{\mathrm{BS}}=\mathrm{m}_{\mathrm{h}}{ }^{*} 100 / \mathrm{m}_{\mathrm{s}}(\%)
$$

Où $m_{h}=$ masse de la matière grasse obtenue après extraction et $\mathrm{m}_{\mathrm{s}}=$ masse des amandes (préalablement séchées à l'étuve).

On effectue une régression linéaire de la teneur en huile en fonction de la teneur en eau pour chaque température.

\section{Résultats et discussion}

\section{Évolution de la teneur en eau}

L'évolution de la vitesse de perte en eau en fonction du temps de friture des amandes est représentée par les courbes de la figure 1. Ces courbes ont une allure classique de déshydratation des produits végétaux riches en eau et présentent un seul maximum chacune. Elles mettent en évidence trois parties du phénomène :

- une phase initiale rapide au cours de laquelle la vitesse maximale est atteinte (2 minutes);

- une période de régime optimum (entre 2 et 30 minutes) ;

- une décélération lente au cours de laquelle la vitesse tend vers zéro.

La vitesse maximale à $120^{\circ} \mathrm{C}$ est de $2,2 \mathrm{~g} \cdot \mathrm{min}^{-1}$ puis elle baisse pour atteindre au bout de 10 minutes de friture une vitesse de l'ordre de 0,8 g. $\min ^{-1}$ qui varie peu pendant la phase suivante. Après 40 minutes de friture à $120^{\circ} \mathrm{C}$, la teneur en eau des amandes entières passe de 56 à $30 \%$. À $180^{\circ} \mathrm{C}$, la vitesse maximale atteint $8,0 \mathrm{~g} \cdot \mathrm{min}^{-1}$, puis baisse progressivement pour se stabiliser autour de $2 \mathrm{~g} \cdot \mathrm{min}^{-1}$ et au bout 40 minutes de friture, la teneur eau des amandes entières est ramenée à $6 \%$ contre 18 et $12 \%$ respectivement à 140 et $160^{\circ} \mathrm{C}$. L'augmentation de la température de friture permet de réduire le temps nécessaire pour atteindre une teneur en eau donnée. Cette réduction du temps de friture par augmentation de la température de friture ne vérifie pas les observations faites par Pravisani et Calvelo [23] qui ont signalé que le temps de friture était indépendant de la température de I'huile dans l'intervalle de 155 à $200^{\circ} \mathrm{C}$. Cette observation leur permettait de noter que l'augmentation de la température de friture n'est pas toujours bénéfique. Plusieurs travaux traitant de la relation entre la teneur en eau et la durée de friture ont montré que la perte d'humidité est proportionnelle à la racine carrée du temps de friture [5, 24-26]. Dans le cas du séchage de la pulpe $d^{\prime}$ avocat, Merion [17] montre que la teneur en eau varie en fonction de la durée de friture, quelle que soit la température, suivant un modèle exponentiel $\mathrm{TE}=\operatorname{Exp}(b+a t)$.

Les équations de régression du tableau 3 ont été obtenues en appliquant cette équation à nos données. On peut remarquer que l'intersection (b) reste constante alors que la pente $(a)$ varie. La moyenne des intersections est de 4,020 $\pm 0,089$. La pente varie avec la température $T$ de friture selon l'équation de régression suivante :

$$
a=0,073-0,001 T\left(r^{2}=-0,974\right)
$$

En utilisant la moyenne des intersections et en remplaçant $a$ par l'équation de régression, on obtient l'équation globale suivante qui lie la teneur en eau des amandes après friture à la durée et à la température de I'opération de friture :

$$
T E=\operatorname{Exp}(4,020+0,073 t-0,001 T . t)
$$

La figure 2 présente les points expérimentaux et les courbes théoriques découlant de cette équation. On note que l'augmentation de la température de friture de 120 à $180^{\circ} \mathrm{C}$ entraîne la baisse de la teneur en eau d'équilibre de $30 \%$ à $2 \%$. On remarque également que quelques points expérimentaux s'écartent des courbes théoriques, ce qui indiquerait que

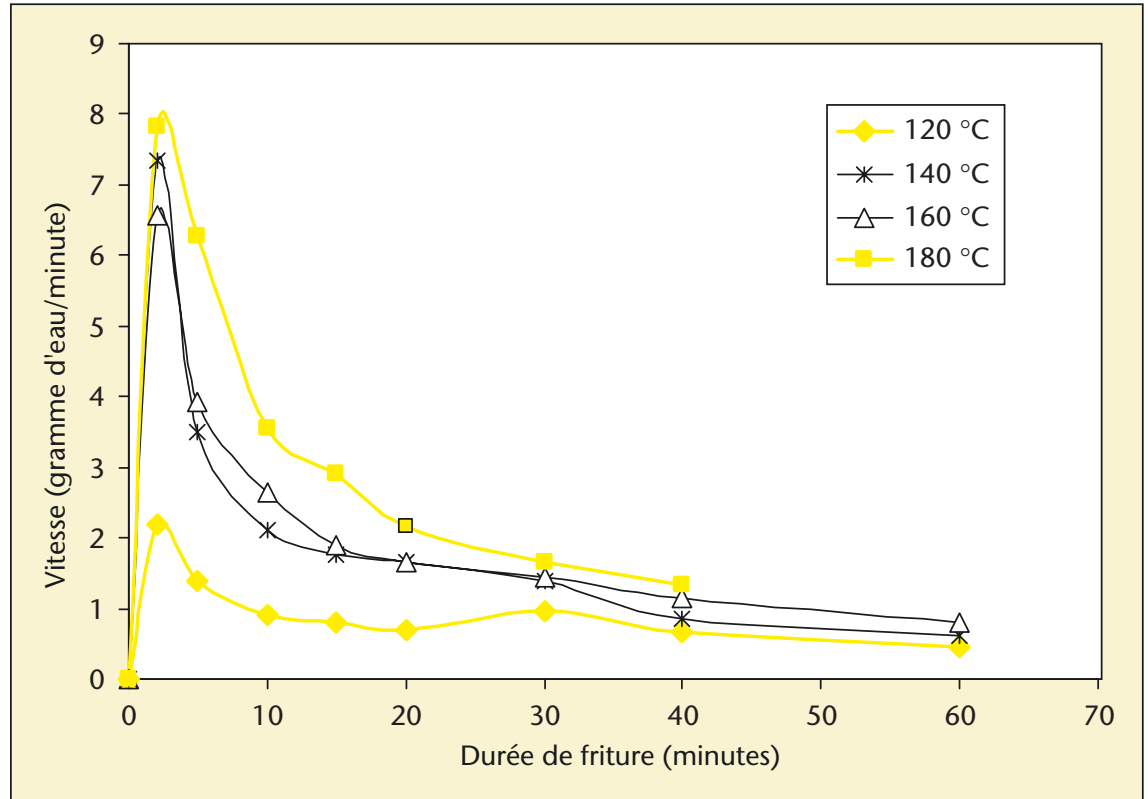

Figure 1. Evolution de la vitesse de séchage des amandes au cours de la friture à différentes températures. 
Tableau 3. Résultats de la régression exponentielle des teneurs en eau.

\begin{tabular}{|lcccc|}
\hline $\begin{array}{l}\text { Température } \\
\left({ }^{\circ} \mathbf{C}\right)\end{array}$ & Equation & $\mathbf{r}^{\mathbf{2}}$ & $\begin{array}{c}\text { Pente } \\
(\boldsymbol{a})\end{array}$ & $\begin{array}{c}\text { Interception } \\
(\boldsymbol{b})\end{array}$ \\
\hline 120 & $\mathrm{TE}=\operatorname{Exp}(4,047-0,011 \mathrm{t})$ & 0,889 & $-0,011$ & 4,047 \\
140 & $\mathrm{TE}=\operatorname{Exp}(3,973-0,026 \mathrm{t})$ & 0,991 & $-0,026$ & 3,973 \\
160 & $\mathrm{TE}=\operatorname{Exp}(3,928-0,034 \mathrm{t})$ & 0,976 & $-0,034$ & 3,928 \\
180 & $\mathrm{TE}=\operatorname{Exp}(4,132-0,055 \mathrm{t})$ & 0,974 & $-0,055$ & 4,132 \\
\hline
\end{tabular}

$T E=$ Teneur en eau. $t=$ temps de friture.

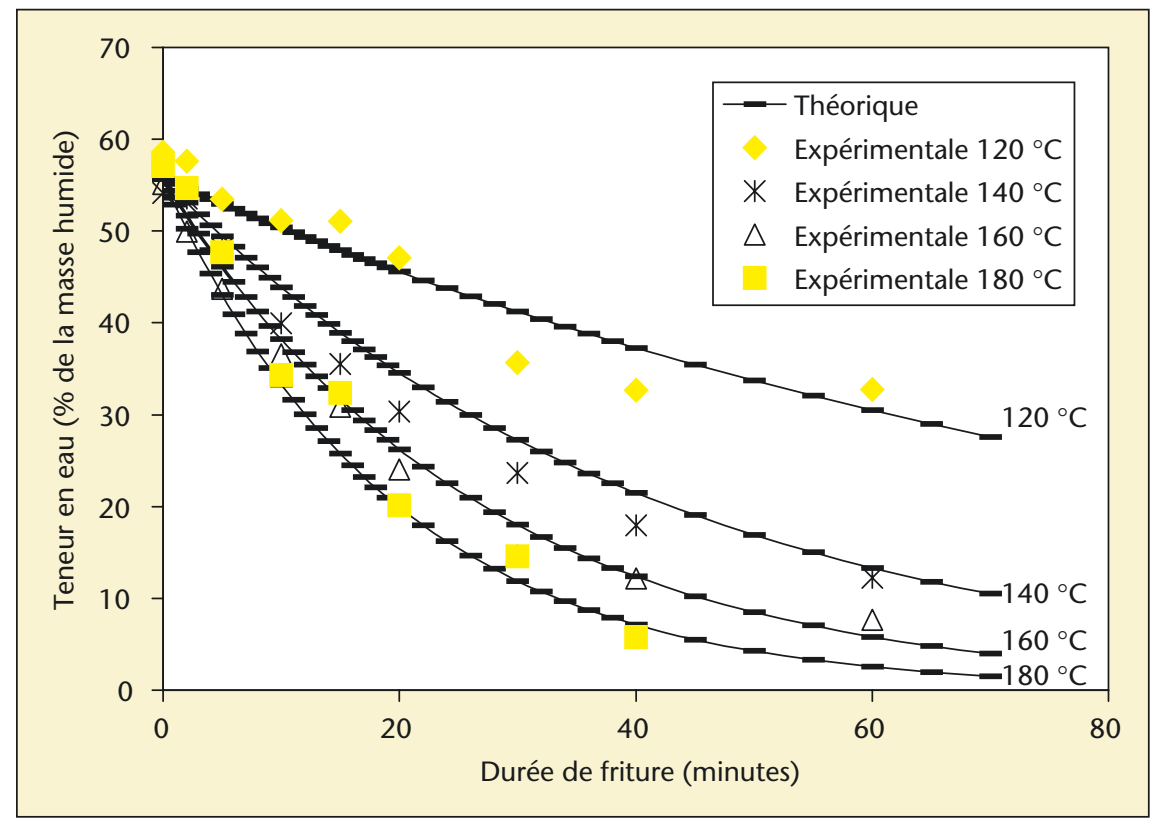

Figure 2. Courbes théoriques et expérimentales de la teneur en eau des amandes au cours de la friture à différentes températures.

d'autres facteurs auraient un effet sur la cinétique de déshydratation des amandes de karité par friture. L'étude des transferts de masse et de chaleur pendant la friture des produits amylacées montre que ces phénomènes de transfert dépendent également de l'épaisseur des tranches et de la teneur en eau du produit [7], ainsi que du rapport massique produit/huile et de la vitesse d'agitation du bain [16]. Ainsi, un affinement du modèle d'expression de la teneur en eau prenant en compte l'épaisseur des amandes et le rapport massique amande/beurre a été conduit selon un plan d'expériences composite centré. Dans ce modèle, l'épaisseur des tranches (e) est exprimée en $\mathrm{mm}$, la température de séchage $(T)$ en ${ }^{\circ} \mathrm{C}$, le temps de friture (t) en minutes et le rapport massique amandes/beurre ( $\mathrm{R}$ ) est adimensionnel. On peut ainsi prédire la teneur en eau (TE) des amandes après friture par l'équation de régression suivante :

$$
\begin{aligned}
& \mathrm{TE}=-66,05+2,99 \mathrm{e}+0,87 \mathrm{~T}+1621,7 \mathrm{R}+3,15 \mathrm{t}-0,09 \mathrm{e}^{2}+ \\
& 0,63 \cdot 10^{-2} \text { e.T21,74e.R+0,04e.t }-0,39 \cdot 10^{-2} \mathrm{~T}^{2}+
\end{aligned}
$$
$L^{\prime}$ analyse du modèle montre que la variation de la teneur en eau dépend à $95,41 \%$ des effets des variables étudiées. Les effets significatifs $(p<0,05)$ sont ceux de l'épaisseur des amandes, de la température de friture, du temps de friture, du carré de l'épaisseur, du carré du rapport massique et de l'interaction entre la température et le temps de friture. Les courbes isoréponses de la figure 3 illustrent la variation de la teneur résiduelle en eau en fonction du temps et de la température de friture. On note que pour ramener la teneur en eau des tranches d'amandes de karité à 10-15\% qui a été signalée comme optimale pour l'extraction [19], il faut les immerger pendant plus de 6 minutes dans un bain de beurre de karité porté à une température supérieure à $140^{\circ} \mathrm{C}$. Ces conditions optimales sont en accord avec les observations faites précédemment sur l'étude cinétique.

Les courbes isoréponses de la figure 4 (variation de la teneur en eau en fonction de l'épaisseur et du temps de friture) montrent que la teneur en eau des amandes optimale pour l'extraction du beurre est obtenue avec les tranches de moins de $12 \mathrm{~mm}$ d'épaisseur, frites pendant plus de 6 minutes. Le rapport amande/beurre (masse/masse) conduisant à cette teneur en eau doit être inférieur à 0,06 pour les durées de friture supérieure à 6 minutes (figure 5). II apparaît difficile d'atteindre de très faibles teneurs finales en eau sur des tranches épaisses, même lorsque le rapport massique devient faible: au bout d'un certain temps de friture il y a formation d'une croûte qui limite l'évaporation de l'eau [27, 28]. En revanche, la friture de tranches fines sous de faibles rapports massiques permet d'atteindre de faibles humidités résiduelles des amandes en peu de temps.

\section{Évolution de la teneur en huile}

La quantité d'huile absorbée par les amandes frites dépend du temps de friture. De plus, une corrélation linéaire négative est observée entre les teneurs en huile et en eau (tableau 4). Plusieurs facteurs ont été rapportés pouvant affecter l'absorption d'huile et les points de vue sont parfois divergents. Par exemple, Fan et Arce [29], mentionnent que, dans un 
Tableau 4. Résultats de la régression linéaire des teneurs en huile (TH) et en eau (TE).

\begin{tabular}{|lcc|}
\hline $\begin{array}{l}\text { Température } \\
\left({ }^{\circ} \mathbf{C}\right)\end{array}$ & Equation & $\mathbf{r}^{\mathbf{2}}$ \\
\hline 120 & $\mathrm{TH}=60,396-0,263 . \mathrm{TE}$ & 0,847 \\
140 & $\mathrm{TH}=65,917-0,384 . \mathrm{TE}$ & 0,882 \\
160 & $\mathrm{TH}=59,872-0.208 . \mathrm{TE}$ & 0,892 \\
180 & $\mathrm{TH}=59,140-0,188 . \mathrm{TE}$ & 0,836 \\
\hline
\end{tabular}

certain intervalle de température donné, l'augmentation de la température de friture réduit l'absorption d'huile, alors que pour Gamble, Rice et Seldman [25] la quantité d'huile absorbée par les pommes de terre est indépendante de la température de friture. Ces mêmes auteurs suggèrent l'existence d'une corrélation linéaire entre la perte en eau et I'absorption d'huile, alors que Du Pont Kirby et Smith [30] ne trouvent pas de relation entre ces deux variables.

\section{Comparaison séchage-friture et séchage convectif}

Le temps de séchage par friture est considérablement réduit par rapport au séchage convectif à l'air. Par exemple, pour atteindre une humidité de $30 \%$ avec des amandes entières fraîches, il faut plus de 60 heures pour un séchage au soleil, une vingtaine d'heures par séchage électrique à convection forcée et seulement 15 à 40 minutes par friture (tableau 5). Cette différence est évidemment liée à la température de séchage : 120 à $180^{\circ} \mathrm{C}$ en friture contre 50 à $60^{\circ} \mathrm{C}$ par séchage électrique et encore moins par séchage au soleil $\left(22\right.$ à $43^{\circ} \mathrm{C}$ observés pendant la saison des pluies). Dans ces conditions de faibles températures et longues durées d'exposition, les lipases restent activent, dégradent les graisses et entraînent ainsi l'augmentation de la teneur en acides gras libres, ce qui détériore la qualité marchande du beurre de karité extrait par la suite.

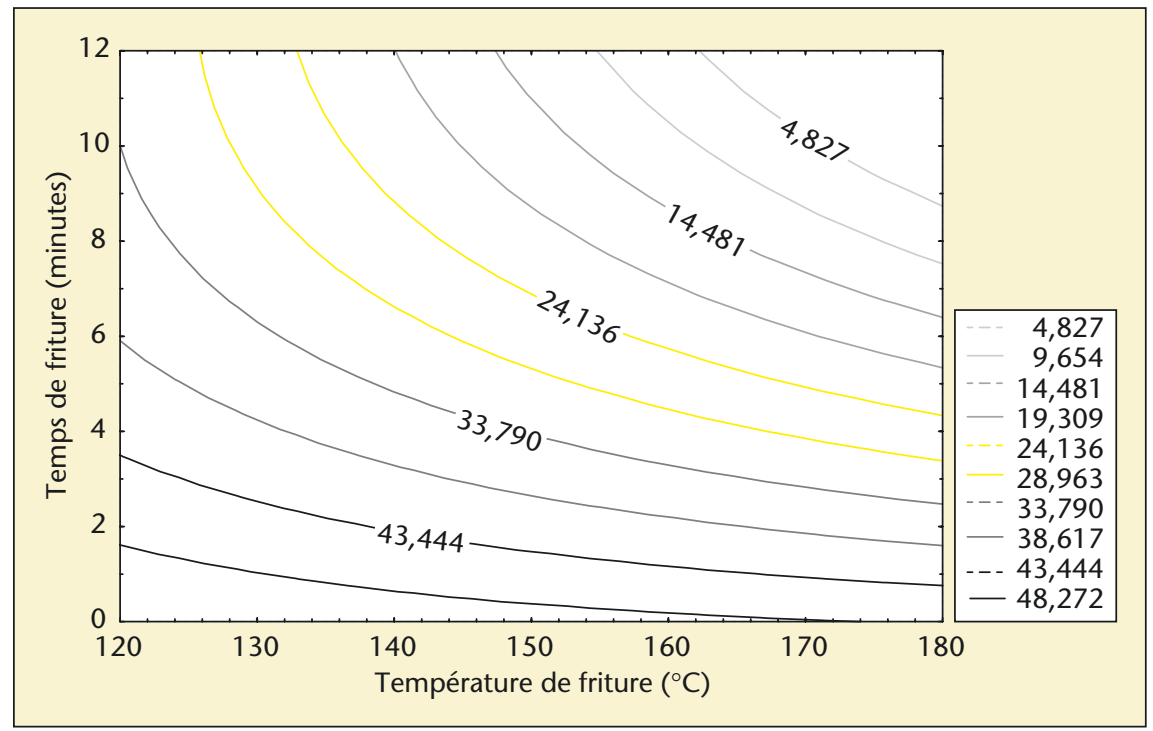

Figure 3. Courbes isoréponses montrant la variation de la teneur en eau résiduelle des amandes (\% de la masse humide) en fonction de la température et du temps de friture.

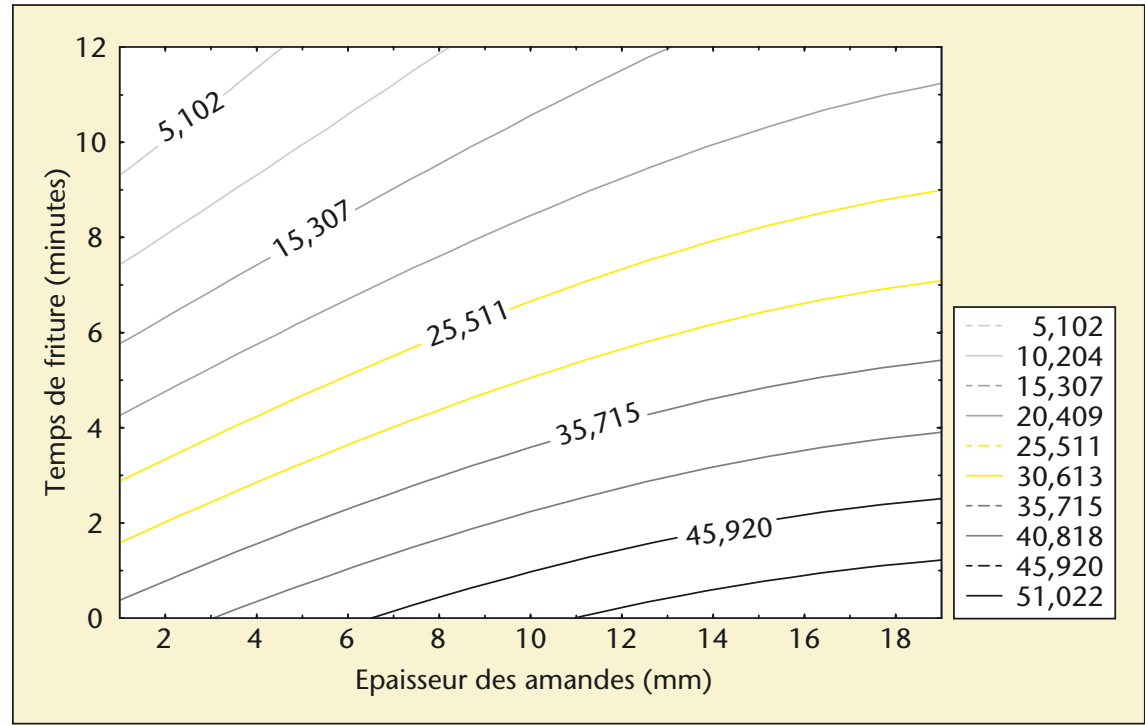

Figure 4. Courbes isoréponses montrant la variation de la teneur en eau résiduelle des amandes (\% de la masse humide) en fonction de l'épaisseur des amandes et du temps de friture. 
Tableau 5. Temps de traitement des amandes de karité nécessaire pour atteindre une humidité de $30 \%$ (base humide) par séchage-friture et séchage convectif.

\begin{tabular}{|lcc|}
\hline Type de séchage & $\begin{array}{c}\text { Température de friture } \\
\left({ }^{\circ} \mathbf{C}\right)\end{array}$ & $\begin{array}{c}\mathbf{t}_{\mathbf{3 0} \%} \\
\text { (minutes) }\end{array}$ \\
\hline Séchage-friture & 120 & 40 \\
& 140 & 20 \\
Séchage convectif à l'air & 160 & 15 \\
& 180 & 15 \\
Séchage solaire direct & 50 & 1 100-1 250 \\
& & $(18$ à 21 heures) \\
& variable & 3 990-4 190 \\
& $\left(22\right.$ à 43 $\left.{ }^{\circ} \mathrm{C}\right)$ & $(66$ à 69 heures) \\
\hline
\end{tabular}

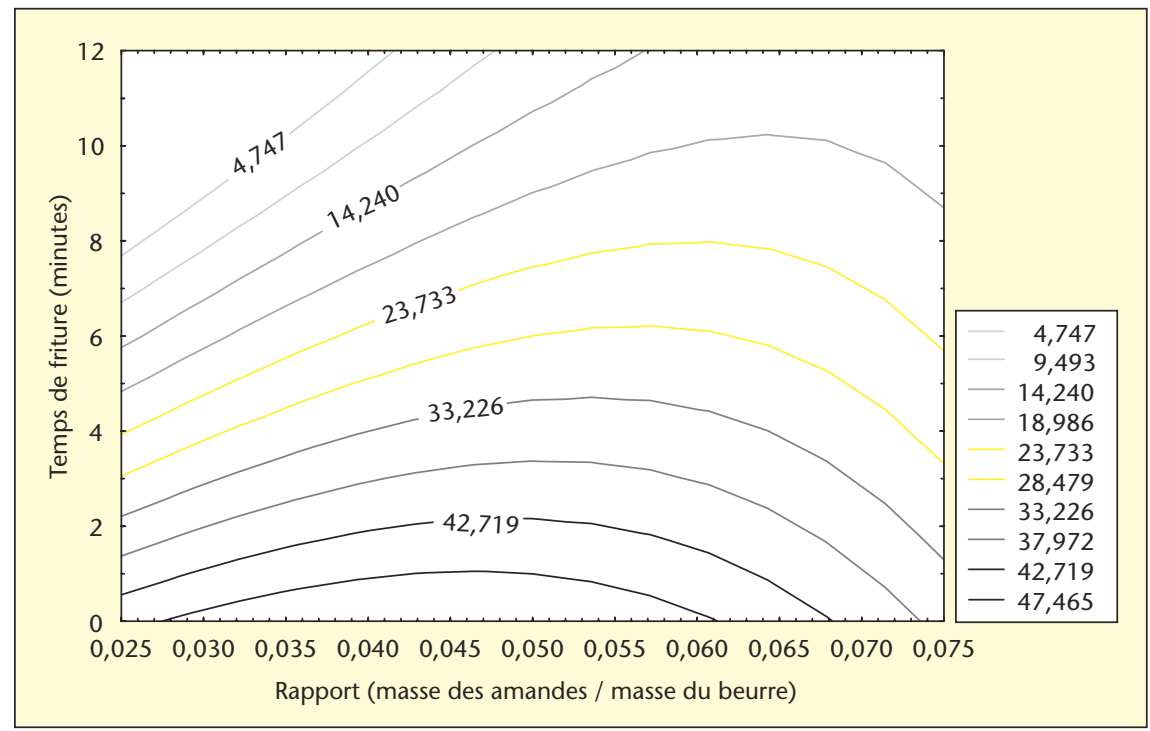

Figure 5. Courbes isoréponses montrant la variation de la teneur en eau résiduelle des amandes (\% de la masse humide) en fonction du rapport massique et du temps de friture.

\section{Conclusion}

Un séchage-friture permet de réduire rapidement et considérablement la teneur en eau des amandes de karité récoltées au Nord-Cameroun. En conditions optimales, des teneurs en eau de 10 à $15 \%$ sont obtenues en séchant pendant 6 minutes des amandes d'épaisseur inférieure à $12 \mathrm{~mm}$, à des températures supérieures à $140{ }^{\circ} \mathrm{C}$, dans un rapport massique inférieur à 0,06. La teneur en eau résiduelle des amandes séchées par friture est prédictible à partir d'un modèle d'équation de régression établi à partir d'un plan d'expériences. Comparé au séchage convectif à l'air (au soleil ou électrique), le temps de séchage est réduit de 30 à 200 fois. Cette étude préliminaire laisse entrevoir une application de ce procédé dans les conditions villageoises dans le but final d'améliorer la qualité marchande du beurre de karité extrait. La recherche des conditions optimales dans l'ensemble des séquences d'extraction devrait permettre d'atteindre la conformité aux cahiers des charges exigeants des clients utilisateurs.

Remerciements. Les auteurs remercient la Fondation internationale pour la science (IFS, Stockholm) et I'Institut de recherche pour le développement (IRD, Paris) pour leur contribution au financement de ce travail.

\section{RÉFÉRENCES}

1. WOMENI HM. Identification et analyse des opérations critiques de préaration des fruits, graines et amandes de karité (Butyrospermum parkii (G. Don) Kotschy): étude de leur influence sur la qualité du beurre. Thèse de Doctorat/PhD en technologie alimentaire, ENSAI, Université de Ngaoundéré, 2004.

2. ASIEDU JJ. La noix de coco. La transformation des produits agricoles en zone tropicale. Approche technologique. CTA - Karthala 1991 : 61-111.

3. DREW PJ, BREAG GR, MARDER RC. Copra production using the coconut shell carbonization with wasteheat recovery technology. Tropical Science 1993 ; 33 : 246-67.

4. RAOULT-WACK AL, LISSE I, ROUZIÈRE A, MONTET D, DUMAS JC, NOËL JM. Séchage de produits gras par friture: Cas des fruits oléagineux (Coco, Avocat) et des sous-produits carnés. Récents progrès en Génie des procédés 1997 ; 11(59) : 69-76 ; vol.

5. RICE P, GAMBLE MH. Modeling moisture loss during potato slice frying. Int J Food Sci Technol $1989 ; 24$ : 183-7.

6. REDDY GV, DAS H. Kinetics of deep-fat-frying of patato and ptimization of process variables. J Food Sci $1993 ; 30(2)$ : 105-8. 
7. BAUMAN B, ESCHER F. Mass and heat transfer during deep-fat-frying of patato slices. I. Rate of drying and oil uptake. Lebensmittel Wissenschaft und Technologie 1995 ; 28(4) : 395-403.

8. COSTA RM, OLIVEIRA FAR, GEKAS V. Application of image analysis to the study of water losses from patato slices during frying. In : Howitt R, ed. Seventh International Congress on Engineering and Food, ICEF97. Sheffied, UK : Sheffield academic Press, 1997 : 157-60.

9. MOREIRA RG, PALAU J, SUN X. Simultaneous heat and mass transfer during the deep fat frying of tortilla chips as a function of frying time. J Food Process Eng $1995 ; 18(3)$ : 307-20.

10. MOREIRA RG, SUN X, CHEN Y. Factors affecting oil uptake in torilla chips in deep-fat-frying. J Food Eng 1997 ; 31 : 485-98.

11. LEMAIRE H, REYNES M, NGALANI JA, GUILLAUMONT A. Aptitude à la friture de cultivars de plantains et bananes à cuire. Fruits 1997 ; 52 : 273-82.

12. DIAZ A, TRYSTRAM G, VITRAC O, DUFOUR D, RAOULT-WACK A. Kinetics of moisture loss and fat absorption during frying for different varieties of plantain. J Sci Food Agric $1999 ; 79$ : 291-9.

13. HAGENIMANA V, OYUNGA M. Oil content in fried sweet patato processed products. In : Akoroda MO, Ekanayake IJ, eds. Proceedings of the sixth triennial symposium of the Inernational Society for Tropia Root Crops-African branch, Lilongwe, Malawi, 22-28 October 1995 Root crops and poverty alleviation. 1998 : 498-502.

14. SOEKARTO ST, MULIAWAN D, ZULVIANI R. (1992). Deep frying expansion of indonesian sago chips (krupuk) as afected by the state of its bound water. In : Singh RP, Wirakartakusumah MA, eds. Advance in Food Engineering. Boca Raton : CRC Press, 1992 : 247-55.

15. KYAW ZY, YU SY, CHEOW CS, DZUKIFLY MH. Effect of steaming time on the linear expansion of fish crakers (Keropok). J Sci Food Agric $1999 ; 79$ : 1340-4.

16. VITRAC O, DUFOUR D, TRYSTRAM G, RAOULT-WACK AL. Characterization of heat and mass tranfer during deep-fat frying and its effect on cassava chip quality. J Food Eng $2002 ; 53$ : 161-76.

17. MERION E. Application du procédé séchage-friture à l'avocat en vue de l'extraction d'huile à usages cosmétiques. Stage de fin d'études, CIRAD/SAR n45/95. Université de Montpellier, 1995.
18. HOUNHOUIGAN I, ROUZIÈRE A, NOËL J, BRICAS N, MAROUZE C, RAOULTWACK AL. Relance de la filière de production d'huile de coco par la technique de séchage friture. Récents progrès en génie des procédés 1997 ; 11(59) : 121-30.

19. WOMENI HM, KAMGA R, TCHIEGANG C, KAPSEU C. Extraction du beurre de karité : influence du séchage des amandes et de la technique d'extraction. La Riv It Sost Gras 2002 ; LXXIX : 33-7.

20. MITTELMAN N, MIZRAHI S, BERK Z. Heat and mass transfer in frying. In : Mckenna BM, ed. Engineering and Foods. London : Elsevier Applied Science, 1982 : 109-16.

21. AFNOR. (Association française pour la normalisation). Recueil des normes françaises. Corps gras, graines oléagineuses, produits dérivés. $2^{e}$ édition. Paris : Afnor, 1981.

22. UIPAC (UNION INTERNATIONALE DE CHIMIE PURE ET APPLIQUÉE). Méthode d'analyse des matières grasses; sixième édition. International Digest of Health Legislation $1979 ; 46: 2$.

23. PRAVISANI Cl, CALVELO A. Minimum cooking time for potato Strip frying. J Food Sci $1986 ; 51: 614-7$.

24. ASHKENAZI N, MIZRAHI S, BERK Z. Heat and mass transfer in frying. In : Singh RP, Heat and Mass Transfer in Foods During Deep-Fat Frying. Food Technol $1995 ; 1984: 134-41$.

25. GAMBLE MH, RICE P, SELDMAN JD. Relationship between oil uptake and moisture loss during frying of potato slices from C.V. Record U.K. tubers. Int J Food Sci Technol 1987 ; 22 : 223-41.

26. KELLER C, ESCHER F. Heat and mass transfer during deep fat frying of potato products. In : RP Singh, Heat and Mass Transfer in Foods During Deep-Fat Frying. Food Technol 1995 ; 49(4) : 134-41.

27. PINTHUS EJ, WEINBERG $P$, SAGUY JS. Oil uptake in deep-fat-frying as affected by porosity. J Food Sci 1995 ; 60 : 767-9.

28. VITRAC O, LISSE I, TRYSTRAM G. Raoult-Wack Al. Séchage par friture, transformations physiques et biochimiques. $16^{\text {es }}$ journées de I'AFSIA, Séchage avec réaction, 28-29 avril 1998, Association Française de Séchage dans I'Industrie et l'Agriculture, 27-35, 1998.

29. FAN LL. Acre JA. Preparation of fried food products with oil containing emulsifiers. US patent $41986 ; 608$ : 264.

30. DU PONT MS, KIRBY AB, SMITH AC. Instrumental and sensory tests of croked frozen french fries. Int J Food Sci Technol 1992 ; 27 : 285-95. 